\title{
Vorwort zur 2. Auflage von Prof. Dr. Rolf Schwartmann
}

In einem stillgelegten und verlassenen Krankenhaus in Büren hatte man augenscheinlich Akten vergessen. Ein Youtuber bekam einen Tipp über den „vergessenen Ort“ und spazierte hinein, um danach ein Video über das Krankenhaus und die Akten zu veröffentlichen. Frei zugängliche offene Krankenakten in einem stillgelegten Krankenhaus sind eine ausgewachsene Datenpanne. Wer haftet? Die seit 2010 insolvente Klinik ist lange geschlossen. Sie steht im Eigentum einer Grundstücksgesellschaft, die einer KlinikenAG gehört. Diese weist die Verantwortung für die Datenpanne von sich und verweist auf den Insolvenzverwalter. Er sei für die sichere Verwahrung ordnungsgemäße Entsorgung und Lagerung der Krankenakten im Inventar des Krankenhauses verantwortlich.

Der Fall ist außergewöhnlich und spektakulär, aber er macht eines deutlich: Als Insolvenzverwalter sollte man den Datenschutz im Auge haben. Dabei macht das vorliegende Werk von Christian Weiß und Nico Reisener deutlich, dass der Datenschutz in der Insolvenz weit vor den Problemen ins Spiel kommt, die in Büren prominent geworden sind. Allen, die sich beruflich mit diesem Bereich befassen, kann ein Blick in das Buch helfen. Es arbeitet die Naht- und Verbindungsstellen des Insolvenzrechts zur DS-GVO heraus und leitet praxisorientiert durch die Materie. Es erklärt Hintergründe, legt den Fokus mit Hilfe von Beispielsfällen auf die Probleme der Praxis und bietet Antworten auf Fragen des Rechtsanwenders.

Köln, im August 2020

Prof. Dr. Rolf Schwartmann Vorsitzender der Gesellschaft für Datenschutz und Datensicherheit (e. V.) 
